\title{
Nanoindentation and nanofriction on DLC films
}

\author{
S. BEC*, A. TONCK and J. FONTAINE
}

LTDS, UMR 5513 CNRS/ECL/ENISE, Ecole Centrale de Lyon, ECULLY, FRANCE

\begin{abstract}
Diamond-like carbon (DLC) coatings have been studied for many years as wear-resistant and low friction materials. Their tribological behaviour depends on the nature of the coating, which is determined by the deposition process. It is also strongly affected by environmental conditions.

In this study, the nanomechanical properties and the nanofriction behaviour of two a$\mathrm{C}: \mathrm{H}$ coatings deposited on silicon substrate and exhibiting different macroscopic friction behaviours were investigated using a three-axial surface force apparatus equipped with a diamond tip.

The hardness, Young's modulus and Poisson's ratio of the two coatings were determined. Viscous recovery of the indents was observed a few days after the indentation tests.

Low friction coefficients (around 0.06) were measured for the coating which exhibited macroscopic low friction. For the coating which exhibits a higher friction coefficient, the dissipative behaviour measured during nanofriction test was enhanced by sliding, compared to the dissipative behaviour measured during normal indentation, suggesting a link between normal viscous dissipation at nanoscale and high friction.
\end{abstract}

Keywords: DLC films; Nanoindentation; Nanofriction; Poisson's ratio; Mechanical properties; Viscous properties

\section{Introduction}

Amorphous forms of pure and hydrogenated carbon - usually named 'Diamond-Like Carbon' or DLC - are of increasing use as protecting coatings in industrial parts, especially in moving mechanical assemblies. These coatings indeed combine high hardness and elasticity, chemical inertness, corrosion resistance and moreover, interesting tribological behaviour, namely low coefficients of friction and low wear. However, the variety of chemical and physical vapour 
deposition techniques and the large range of process parameters permit the synthesis of many compositions and film's structures making it possible to control the physical and mechanical properties of a DLC film. Recent reviews describe the various structures and properties of DLC coatings and some of their applications [1]. Their macroscopic tribological behaviour has been also widely studied, and many reviews can be found in the literature $[2,3]$.

Among the various structures of DLC materials, hydrogenated amorphous carbon (a-C:H) films are of special interest, since some of these films may exhibit extremely low friction coefficients in high vacuum [4] or inert environments [5] for macroscopic contacts (contact diameter from few tens to several hundreds of micrometers). With barely measurable values - typically lower than 0.01 - this vanishing of sliding resistance is often referred to as 'super-low friction' or 'super-lubricity'. Experimental evidence of such phenomena for macroscopic contacts has been first reported in the early nineties for very pure molybdenum disulfide coatings $\left(\mathrm{MoS}_{2}\right)$, also under ultra-high vacuum [6]. For this lamellar crystalline material, some theoretical approaches [7] and experimental evidences [8] suggest that incommensurability of contacting surfaces might lead to such friction reduction. More recently, this structural phenomenon has been also evidenced for graphite, another lamellar hexagonal solid, in nano-scale contacts under vacuum [9]. Nevertheless, such crystalline origin of the friction reduction cannot account for the reported values on DLC films, which exhibit amorphous structures. Besides, these very low values of friction coefficient are not achievable with all DLC film: according to the literature, only some hydrogenated amorphous carbon $(\mathrm{a}-\mathrm{C}: \mathrm{H})$ exhibit such friction reduction under vacuum or inert environment, providing their hydrogen content is high enough. Indeed, under high vacuum, the friction coefficient of these films stabilizes either at very low or very high values, depending on hydrogen content, as suggested by many authors $[10,11,12,13]$.

Now, how to identify the mechanisms involved in the super-lubricity of hydrogenated amorphous carbon? Since a carbon-rich transfer film is build-up on many counterfaces when friction and wear are low [3], superlow friction always occurs between two surfaces coated with hydrogenated amorphous carbon, which might nevertheless be different from the original film. The strong differences observed under ultra-high vacuum between high and low friction films could be explained by surface interactions between the carbon-covered counterfaces. For higher hydrogen content in the film, surface covering by hydrogen atoms might lead to weak interactions between the sliding surfaces, whereas for lower hydrogen content, the strong interactions between the $\pi$ orbitals of $\mathrm{sp}^{2}$ carbon double bonds become predominant $[14,15]$. Moreover, the high friction under ultra-high vacuum can be avoided by the 
introduction of gaseous hydrogen, probably by promoting tribochemical reactions between hydrogen and the a-C:H surface $[16,17]$. Thus, the lubricating role of hydrogen on a-C:H surface is now commonly accepted, with weak Van-der-Waals interactions accounting for the super-low friction values.

In spite of these results, complete understanding of the super-low friction of a-C:H films is not yet achieved. Indeed, it was shown in previous studies that the reported threshold in hydrogen content between high and low friction strongly depend on the deposition process [18]. By comparing samples from three different plasma enhanced chemical vapour deposition (PECVD) processes, threshold was found to vary between 26 and 46 at.\%. Thus, surface coverage by hydrogen should vary greatly between several super-low friction coatings. In this previous work, it was also shown that achievement of super-lubricity for a-C:H coatings was correlated to viscoplasticity.

The aim of the present paper is to study, at nanometric scale, the mechanical and tribological behaviour of two a-C:H samples, one above and one below the threshold hydrogen content, in order to improve our understanding of the super-lubricity of such amorphous material in multi-asperity macro-scale contacts.

\section{Experimental details}

\subsection{DLC samples}

Two typical hydrogenated amorphous carbon samples with different hydrogen contents were compared in this study. These coatings were obtained by d.c. plasma enhanced chemical vapour deposition (d.c.-PECVD)[19]. For the two samples, the precursor gas was acetylene $\left(\mathrm{C}_{2} \mathrm{H}_{2}\right)$, with two different couples of bias voltage on the electrodes and pressure in the deposition chamber. The sample labelled AC8 was obtained at $-800 \mathrm{~V}$ under $13 \mathrm{~Pa}$, while the sample labelled AC5 was obtained at $-500 \mathrm{~V}$ under $26 \mathrm{~Pa}$. These different conditions will modify the composition and structure of the coatings. Deposition times were adjusted to achieve a thickness of about $1 \mu \mathrm{m}$ for both AC8 and AC5 coatings on mono-crystalline (100) silicon substrates.

These samples were characterized in previous studies with different techniques in order to determine their composition and structure $[13,20]$, as well as some mechanical and tribological properties [18]. The evolution of the coefficient of friction was recorded under ultra-high vacuum $\left(<10^{-6} \mathrm{~Pa}\right)$ for linear reciprocating contacts against 52100 bearing steel 
pins of $8 \mathrm{~mm}$ curvature radius, under $3 \mathrm{~N}$ load - leading to a theoretical maximum Hertzian pressure of about $450 \mathrm{MPa}$ - for up to 500 cycles. Hardness and Young's modulus were measured by nanoindentation with a Berkovich tip with maximum load of $100 \mathrm{mN}$, and by applying the load exponentially versus time, in order to maintain a constant strain rate during indentation. The evolution of hardness, studied for strain rates between 0.003 and $0.3 \mathrm{~s}^{-1}$, followed a Norton-Hoff law: $H=H_{0} \cdot \dot{\epsilon}^{x}$, where $H$ is the hardness, $H_{0}$ a constant, $\dot{\epsilon}$ the strain rate and $x$ the viscoplastic exponent.

All the relevant data from previous work on these coatings are gathered in Table 1.

\subsection{Surface force apparatus}

The previous study by nanoindentation gave mechanical information in a depth range of $100 \mathrm{~nm}$ to $1 \mu \mathrm{m}$, revealing the average mechanical properties of the coatings. Since the coatings are about $1 \mu \mathrm{m}$ thick, the measured elastic properties were thus significantly affected by the substrate. In this study, a surface force apparatus is used to have access to surface mechanical properties of the coatings, with minor effect of the substrate on elastic response.

The Ecole Centrale de Lyon three-axial surface force apparatus (also named '3D2F') used in this study has been precisely described in previous publications [21]. A picture of the device is shown on figure 1. The general principle is that a macroscopic spherical body or a diamond tip can be moved toward and away from a planar one using the expansion and the vibration of a piezoelectric crystal along the three directions, Ox, Oy (parallel to the plane surface) and $\mathrm{Oz}$ (normal to the plane surface). The plane specimen is supported by double cantilever sensors, measuring normal $F_{Z}$ and tangential $F_{X}$ forces. The sensor's high resolution $(10 \mathrm{nN})$ allows a very low compliance to be used for the force measurement (up to $2.10^{-6} \mathrm{~m} / \mathrm{N}$ ). Three capacitive sensors are used to measure relative displacements in the three directions between the supports of the two solids, with a resolution of $0.01 \mathrm{~nm}$ in each direction. Experiments are conducted either by imposing a displacement to the sphere (or tip) and measuring the resulting forces, or by imposing a force to the sphere (or tip) and measuring the resulting displacement. Dynamic measurements are simultaneously obtained by adding small vibrations (few angstroms amplitude and at a frequency of $37 \mathrm{~Hz}$ in the normal direction and $70 \mathrm{~Hz}$ in the tangential one) to the quasi-static slow movement both in normal and tangential directions. Thus normal $K_{z}$ and tangential $K_{X}$ stiffnesses, related to normal and tangential elastic behaviours, are simultaneously obtained from the in-phase signals, and the 
out of phase signals, $\omega A_{z}$ and $\omega A_{x}$, are related to the dissipative phenomena, such as viscous damping.

This instrument permits several kinds of experiments performed in controlled atmosphere: nanorheology experiments and nanofriction tests with the sphere/plane geometry or nanoindentation, nanoscratch and nanofriction tests with the tip/plane geometry [21]. In this study, the apparatus was equipped with a Berkovich diamond tip (angle $115.12^{\circ}$ between edges) and used to perform nanoindentation tests and nanofriction experiments, in dry argon atmosphere.

\section{Results and discussion}

\subsection{Surface mechanical properties}

Typical nanoindentation curves, obtained at a constant indentation speed of $0.5 \mathrm{~nm} / \mathrm{s}$ on AC8 and AC5 a-C:H samples, are plotted on figure 2, for maximum loads of 1000 and $2000 \mu \mathrm{N}$. These curves reveal a high elastic recovery. However, as can be seen in the inset on figure 2, a persistent indent is clearly visible after the experiment, evidencing a plastic deformation. Nevertheless, some relaxation occurs, since comparison of the same indent on AC8 within minutes and 6 days after indentation at $2000 \mu \mathrm{N}$ reveals some significant recovery (figure 3), with the maximum depth decreasing from $23 \mathrm{~nm}$ down to $15 \mathrm{~nm}$. This result is consistent with the viscoplastic nature of these amorphous materials [18].

Despite some dispersion between curves obtained at different positions on the same sample, AC8 sample exhibits significantly higher mechanical properties than AC5 sample, which has higher hydrogen content. Mechanical properties can be evaluated by using the load/displacement curves together with the normal contact stiffness $K_{z}$, using a previously described method [22]. The hardness $H$ of the films is directly obtained as the maximum indentation depth is near $10 \%$ of the film thickness. For the reduced Young's modulus $E^{*}$, the contribution of the diamond indenter to the elastic response is first substracted, and then the contribution of the substrate can be deducted using a simple model previously described [22], although it has here a quite small effect for lowest indentation depths. Finally, AC8 film has a hardness of $10.6 \pm 1 \mathrm{GPa}$ and a film reduced Young's modulus of $80 \pm 5 \mathrm{GPa}$, while for AC5 film the values are $7.5 \pm 0.6 \mathrm{GPa}$ and $62 \pm 4 \mathrm{GPa}$ respectively. Thus, both films exhibit relatively low $E^{*} / H$ ratios (around 8 ), as suggested by the strong elastic recovery observed on their indentation curves. Therefore indenting hydrogenated amorphous carbon films with a cube-corner tip (with $90^{\circ}$ angle) would probably be better to promote plastic deformation, 
since it will impose a higher strain compared to Berkovich tip [23]. Nevertheless, the persistence of indents several days after experiment authorizes the use of an elasto-plastic model to compute the mechanical properties of these films.

Furthermore, thanks to the simultaneous measurement of normal and tangential contact stiffnesses, $K_{z}$ and $K_{x}$, the Poisson's ratio $v$ can also be estimated. From figure 4a, which displays the evolution of tangential stiffness $K_{x}$ as a function of normal stiffness $K_{z}$ for experiments on AC8 and AC5 with smallest scattering, it is observed that at this scale (plastic indentation depth of about $5 \%$ of the film thickness), these DLC films behave like isotropic bulk material as the ratio $K_{x} / K_{z}$ is between $2 / 3$ and 1 . The same curve obtained for bulk silicon using the same technique is plotted as reference. In this case, the measured tangential and normal stiffnesses are respectively related to the reduced shear modulus $G^{*}$ and the reduced Young's modulus $E^{*}$ according to the following equations: $K_{x}=8 G^{*} a$ [23] and $K_{z}=2 E^{*} a[24,25]$, where $a$ is the equivalent contact radius. Since we consider a contact between a diamond tip and a DLC sample, we should consider both shear and Young's moduli as composite ones, with the following expressions, where 1 refers to the sample and 2 to the tip:

$$
\frac{1}{G^{*}}=\frac{2-\nu_{1}}{G_{1}}+\frac{2-\nu_{2}}{G_{2}} \text { and } \frac{1}{E^{*}}=\frac{1-\nu_{1}^{2}}{E_{1}}+\frac{1-\nu_{2}^{2}}{E_{2}}
$$

However, the values of Young's modulus measured for our samples are lower than $90 \mathrm{GPa}$, compared to around $1000 \mathrm{GPa}$ for the diamond tip. On the other hand, the following relation between $G$ and $E$ must be considered:

$$
G=\frac{E}{2(1+\nu)}
$$

Thus, contributions of diamond to both $G^{*}$ and $E^{*}$ can be neglected. Finally, the ratio between tangential and normal stiffnesses can be related to Poisson's ratio $v$ of the DLC samples in the following way:

$$
\frac{K_{x}}{K_{z}}=\frac{2(1-\nu)}{2-\nu}
$$

Poisson's ratio $v$ can thus be calculated from these data, and the resulting distribution of values during indentation is reported on figure $4 \mathrm{~b}$. For bulk silicon, an average value of 0.25 is achieved, in good agreement with values found in the litterature [26]. For the hydrogenated amorphous carbon films, especially AC5, more widely distributed values are found. This distribution mainly originates from the tangential stiffness. Because almost no scattering was observed under the same conditions on bulk silicon, this high scattering cannot be attributed 
to the experimental setup, but rather to the samples themselves. Hence, only general trend can be deduced from these experiments, by means of a statistical approach. For AC8 sample, half of the values range between 0.15 and 0.25 , with a median value of 0.18 . For AC5 sample, half of the values range between 0.05 and 0.25 , with median value of 0.12 . Nevertheless, the Poisson's ratio values obtained are clearly lower than for silicon, in the range of vitreous silica values [27].

\subsection{Nanofriction}

The '3D2F' surface force apparatus also permits force/displacement measurements along the tangential direction. Thus, it is possible to perform friction experiments at nanometric scale. These tests were performed with the same diamond Berkovich tip at different normal loads from $10 \mu \mathrm{N}$ to $300 \mu \mathrm{N}$. The sliding speed varied from 0.2 to $100 \mathrm{~nm} / \mathrm{s}$ and the sliding distance was $1 \mu \mathrm{m}$. AC8 and AC5 hydrogenated amorphous carbon films appear quite rough at this scale, as can be seen on the inset of figure 2 or on figure 3 . In such case, the contribution of the topography to the tangential force $F_{X}$ becomes significant, especially at low loads. Therefore, reciprocating motion was performed, and the coefficient of friction $\mu$ was computed in the following way:

$$
\mu=\frac{F_{\bar{\Sigma}}-F_{\mathrm{x}}^{\infty}}{2 F_{\mathrm{g}}}
$$

where $F_{x}^{\leftarrow}$ and $F_{x} \rightarrow$ are the tangential forces for respectively back and forth motion. Figure 5 displays the tangential force $F_{X}$ as a function of tangential displacement $X$ for a test on AC5 sample at a normal load of $11.5 \mu \mathrm{N}$. With this method, the coefficient of friction is about 0.06 for AC5 and about 0.12 for AC8. It is noticeable that AC5 sample exhibits the lowest friction and had the highest scattering on $K_{x}$ measurements, resulting in the strongest dispersion of Poisson's ratio values. This might come from some sliding during indentation due to the tangential vibration.

The coefficient of friction is higher for AC8 than for AC5, as it was observed for the macroscopic stabilized coefficients of friction measured under ultra-high vacuum in previous studies (see table 1), although a factor of only 2 separates both samples instead of more than 100. Several reasons might account for such difference. Gaseous environment - argon instead of vacuum - and nature of antagonist - diamond instead of carbonaceous tribofilm on steel are indeed different and might lead to different interactions between contacting surfaces. 
Furthermore, the nanofriction tests presented here correspond to the first friction cycle and not to stabilized friction after hundreds of cycles.

Nevertheless, in order to further investigate this difference between friction coefficients, the viscous behaviour of the two samples during friction was compared. This was done by looking at the out of phase signals from the dynamic measurements, $\omega A_{z}$ and $\omega A_{x}-$ related to the dissipative phenomena such as viscous damping - which were recorded simultaneously to the in-phase signals related to elastic behaviour $\left(K_{z}\right.$ and $\left.K_{x}\right)$. The normal viscous damping $\omega A_{z}$ measured for AC8 was found to increase during friction, compared to the value measured during normal indentation as shown on figure 6. On this curve, the maximum viscous damping measured in the normal direction during friction at a constant normal load of $100 \mu \mathrm{N}$ (mark A on figure 6) is about twice the maximum viscous damping during normal indentation (marks $\mathrm{B}$ on figure 6 for loading and $\mathrm{C}$ for unloading). Even if such experiments are extremely delicate to conduct, this effect is significant and was not observed for AC5, with viscous damping during friction comparable to viscous damping during normal indentation. This result may explain the difference of friction coefficient observed between AC8 and AC5 at this scale: higher viscous damping leads to higher energy dissipation and then higher friction force. Such phenomenon is probably paramount at this scale, since the coating's properties play a major role in the mechanical response, as opposed to macroscopic scale, where the substrate's properties influence significantly the mechanical behaviour of the system. The important viscous damping observed in these experiments probably prevents superlow friction to be achieved with AC5 at nanoscale.

\section{Conclusion}

Local mechanical properties of two hydrogenated amorphous carbon samples were measured using a surface force apparatus. The DLC film with the highest hydrogen content (AC5) exhibits the lowest hardness and Young's modulus. Thanks to tangential and normal vibrations added to the quasi-static indentation, an original method allowed us to estimate the Poisson's ratio of such amorphous DLC coatings. The values obtained are clearly lower than 0.2 , despite significant dispersion.

The coefficient of friction was also measured at nanometric scale on these samples. AC5 sample achieved a low friction coefficient of 0.06 , half of AC8 value. For AC8, it was also observed that the normal dissipative behaviour was enhanced by sliding compared to normal indentation. This can explain the difference in friction coefficient between the two 
films. This study illustrates moreover the difficulty in tribology to correlate local phenomena to macroscopic behaviour, since different contributions are involved depending on the investigation scale.

\section{Acknowledgments}

The authors would like to thank Dr. Alfred Grill from IBM T.J. Watson Research Center for providing AC5 and AC8 samples.

\section{References}

[1] J. Robertson, Mater. Sci. Eng. R 37129 (2002).

[2] A. Grill, Surf. Coat. Technol. 94-95(1-3) 507 (1997).

[3] A. Erdemir, and C. Donnet, Modern Tribology Handbook, vol. 2, edited by B. Bhushan (Boca Raton, CRC Press, 2000), pp. 871-908.

[4] C. Donnet, M. Belin, J.C. Augé, J.M. Martin, A. Grill, and V. Patel., Surf. Coat. Technol. 68-69 626 (1994).

[5] A.Erdemir, O.L. Eryilmaz and G. Fenske, J. Vac. Sci. Technol. A 18(4) 1987 (2000).

[6] J.M. Martin, C. Donnet, T. Le Mogne and T. Epicier, Phys. Rev. B 4810583 (1993).

[7] K. Shinjo and M. Hirano, Surface Science 283473 (1993).

[8] J.M. Martin, H. Pascal, C. Donnet, T. Le Mogne and J.L. Loubet, Surf. Coat. Technol. 68-69 427 (1994).

[9] M. Dienwiebel, N Pradeep, G.S. Verhoeven, H. Zandbergen and J.W.M. Frenken, Surface Science 576197 (2005)

[10] S. Miyake, S. Takahashi, I. Watanabe and H. Yoshihara, ASLE Trans. 30(1) 21 (1987).

[11] H. Zaidi, T. Le Huu and D. Paulmier, Diamond Relat. Mater. 3787 (1994).

[12] T. Le Huu, H. Zaidi and D. Paulmier, Wear 181-183 766 (1995).

[13] C. Donnet and A. Grill, Surf. Coat. Technol. 94-95 456 (1997).

[14] C. Donnet, A. Grill, J. Fontaine, T. Le Mogne, F. Lefebvre, V. Patel and C. Jahnes. Lubrication at the frontier, Tribology Series 36, edited by D. Dowson et al. (Elsevier, Amsterdam, 1999), pp. 333-341.

[15] A. Erdemir, Tribol. Internat. 371005 (2004).

[16] C. Donnet, J. Fontaine, A. Grill and T. Le Mogne, Tribol. Lett. 9 (3-4) 137 (2000).

[17] J. Fontaine, C. Donnet, A. Grill and T. Le Mogne, Surf. Coat. Technol. 146-147 286 
(2001).

[18] J. Fontaine, J.L. Loubet, T. Le Mogne and A. Grill, Tribol. Lett. 17(4) 709 (2004).

[19] A. Grill and V. Patel, Diamond Films Technol. 1219 (1992).

[20] C. Donnet, J. Fontaine, F. Lefebvre, A. Grill, V. Patel and C. Jahnes, J. Appl. Phys. 85(6) 3264 (1999).

[21] A. Tonck, S. Bec, D. Mazuyer, J. M. Georges, A. A. Lubrecht, Proc. Instn. Mech. Engrs. 213353 (1999).

[22] S. Bec, A. Tonck, J. M. Georges, E. Georges and J. L. Loubet, Phil. Mag. A 74(5) 1061 (1996).

[23] K.L. Johnson, in Contact Mechanics (Cambridge University Press, Cambridge, 1985).

[24] I.N. Sneddon, International Journal of Engineering Science, 347 (1965).

[25] J.L. Loubet, J.M. Georges, G. Meille, Microindentation Techniques in Materials Science and Engineering, ASTM STP 889, edited by P.J. Blau and B.R. Lawn (American Society for Testing and Materials, Philadelphia, 1986), pp. 72-89.

[26] D.R. França and A. Blouin, Meas. Sci. Technol. 15859 (2004).

[27] J. Perez, in Matériaux non cristallins et science du désordre (Presses Polytechniques Universitaires Romandes, Lausanne, 2001). 


\section{Table caption}

\section{Table 1}

Deposition parameters, composition and macroscopic mechanical and tribological properties of the two DLC samples (from $[13,18,20]$ ).

\section{Figure caption}

\section{Figure 1}

Schematic drawing of the three-axial Surface Force Apparatus '3D2F' of the Ecole Centrale de Lyon.

\section{Figure 2}

Typical indentation curves obtained at $1000 \mu \mathrm{N}$ (full symbols) and $2000 \mu \mathrm{N}$ (open symbols) for the two DLC films : black circles for AC8 and grey triangles for AC5.

In inset, topographic image of a $2000 \mu \mathrm{N}$ load indent on AC8 obtained with the same diamond tip and device by scanning the surface at imposed low normal force $(1 \mu \mathrm{N})$.

\section{Figure 3}

Images of indents obtained on AC8 at $2000 \mu \mathrm{N}$ load

(a) a few minutes after the indentation test

(b) six days after the indentation test

Color changes are related to height variation with each color change corresponding to a height variation of $1 \mathrm{~nm}$.

\section{Figure 4}

(a) Evolution of the tangential contact stiffness $K_{X}$ versus normal contact stiffness $K_{z}$ for AC8, AC5 and bulk silicon for comparison. Straight lines $K_{x}=K_{z}$ and $K_{x}=2 / 3 K_{z}$ define the domain of isotropic bulk materials.

(b) Distribution of the values of Poisson's ration for AC8, AC5 and silicon for comparison. 


\section{Figure 5}

Friction force $F_{X}$ versus tangential displacement $X$ measured for AC5 during one friction cycle at a constant normal load of $11.5 \mu \mathrm{N}$.

\section{Figure 6}

Evolution of the normal viscous damping $\omega A_{z}(\bullet)$ during a friction test on AC8. The evolution of the normal load $F_{Z}(\square)$ and tangential displacement $X(\triangle)$ are plotted to indicate the different stages during the test. Marks A, B and C point out the normal viscous damping measured respectively during sliding, loading and unloading. 


\section{TABLE 1}

AC8 $\quad$ AC5

\begin{tabular}{ccc}
\hline Precursor and pressure & $\mathrm{C}_{2} \mathrm{H}_{2}$ at $13 \mathrm{~Pa}$ & $\mathrm{C}_{2} \mathrm{H}_{2}$ at $27 \mathrm{~Pa}$ \\
\hline Bias voltage (d.c.) & $-800 \mathrm{~V}$ & $-500 \mathrm{~V}$ \\
\hline Hydrogen content (at. \%) & $34 \%$ & $40 \%$ \\
\hline $\mathbf{C ~ s p}^{2}: \mathbf{C ~ s p}^{3}$ ratio & $70: 30$ & $65: 35$ \\
\hline $\mathbf{H}$ bonded to $\mathbf{C}$ (NMR / FTIR) & $93 \% / 57 \%$ & $98 \% / 73 \%$ \\
\hline Hardness $\boldsymbol{H}_{0}$ (GPa) & 13 & 11 \\
\hline Viscoplastic exponent $\boldsymbol{x}$ & 0.014 & 0.068 \\
\hline $\begin{array}{c}\text { Stabilized coefficient of friction } \\
\text { in ultra-high vacuum }\end{array}$ & 0.53 & 0.003 \\
\hline
\end{tabular}




\section{FIGURE 1}

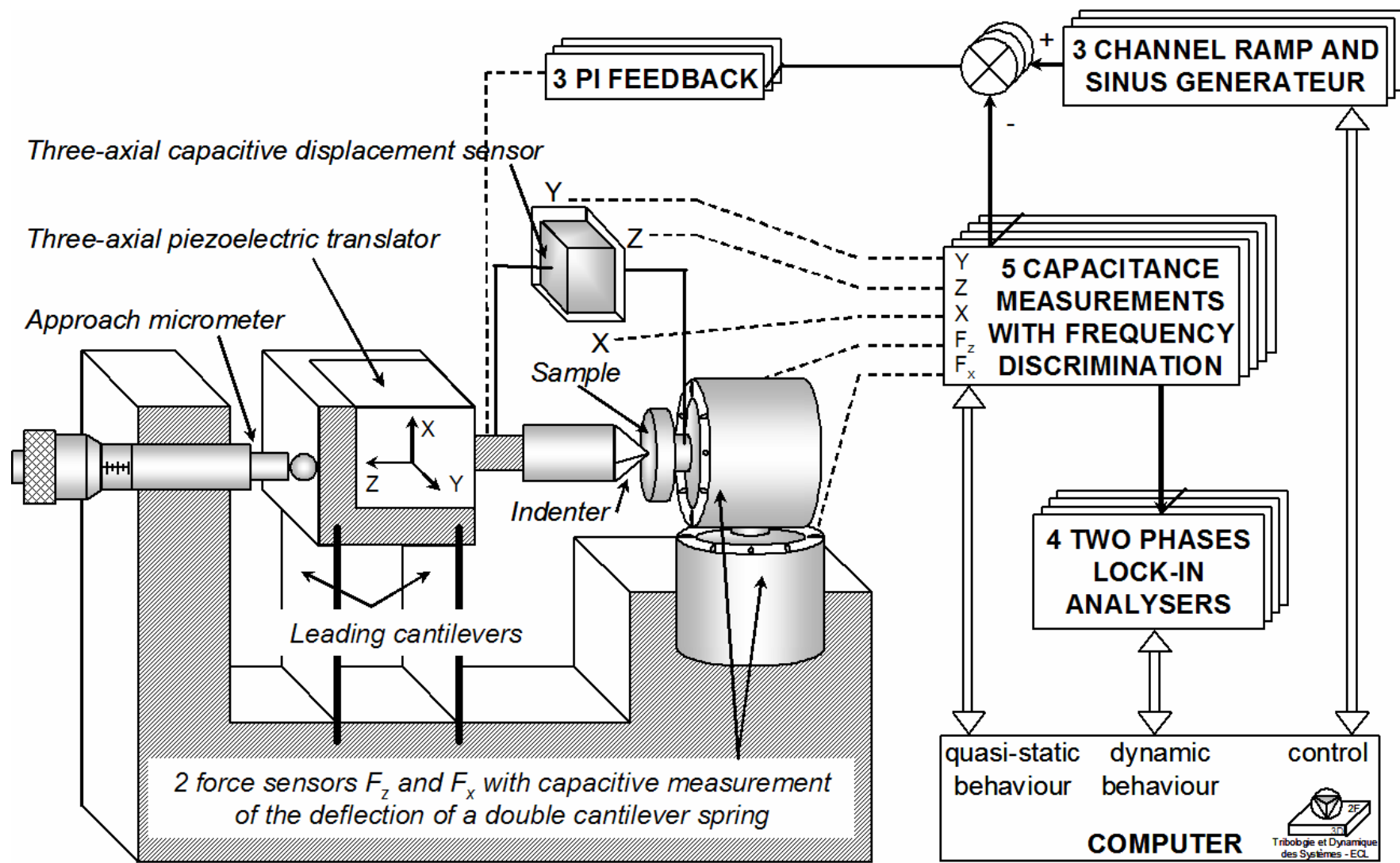




\section{FIGURE 2}

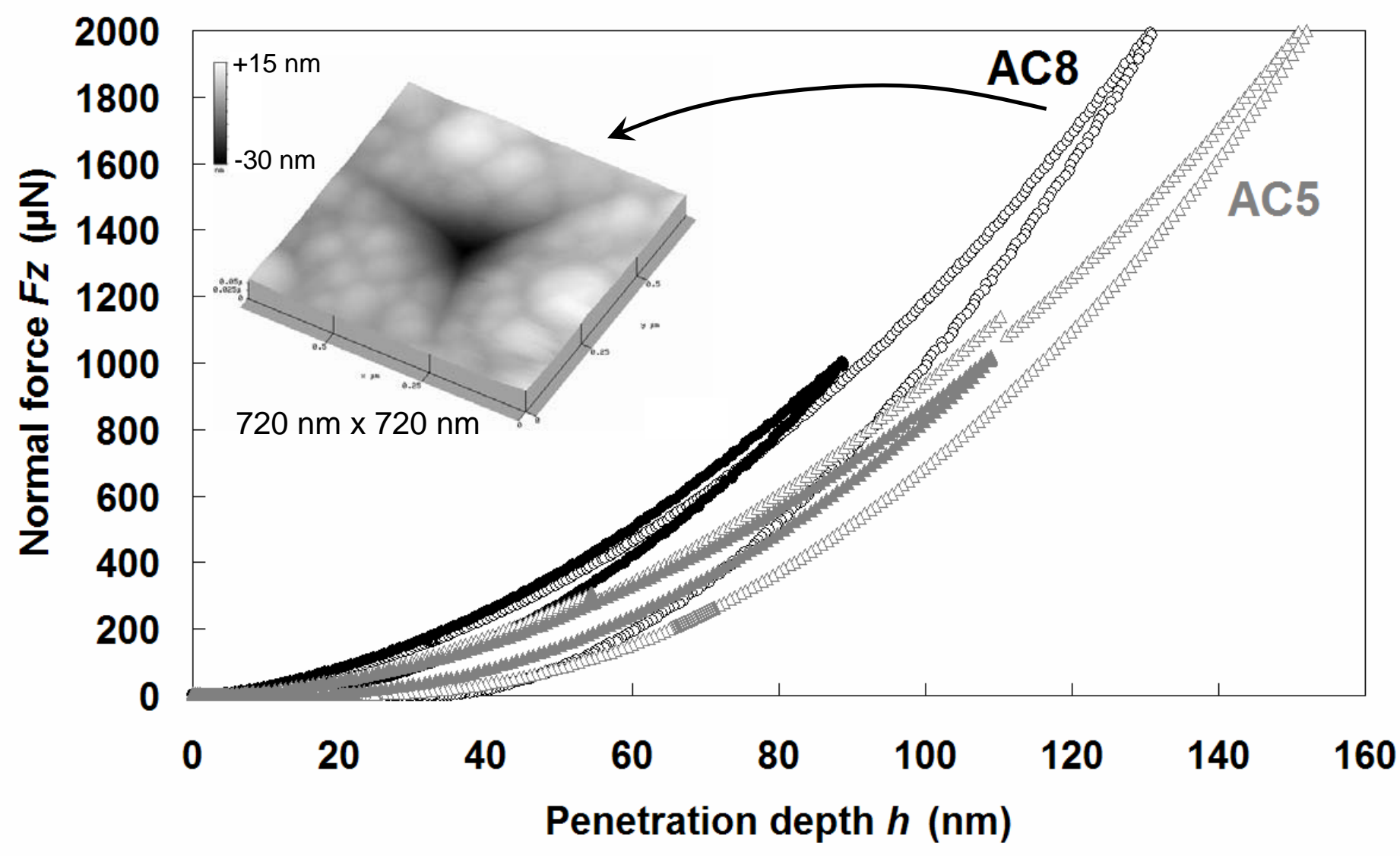




\section{FIGURE 3}

(a)

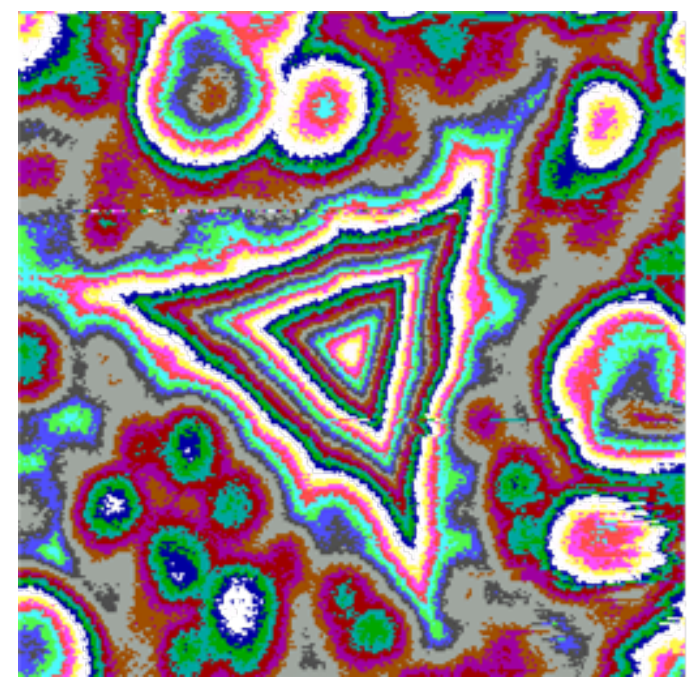

(b)

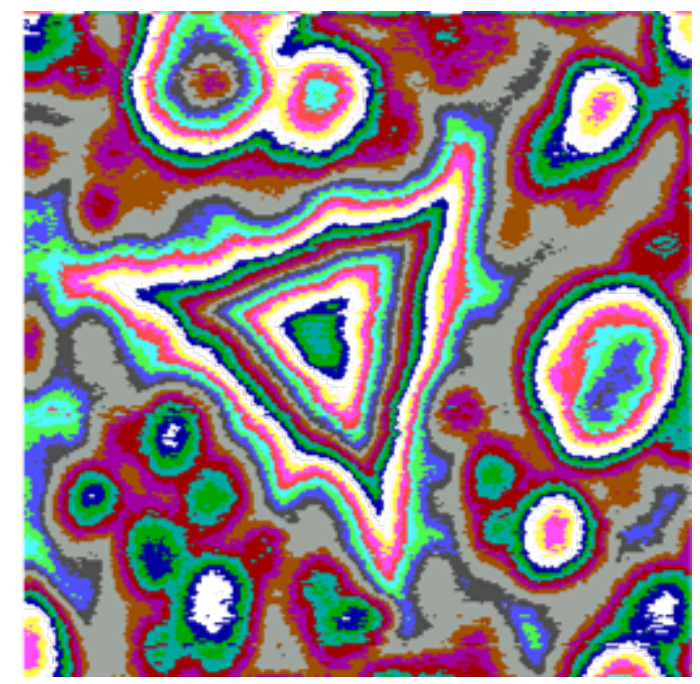

$720 \mathrm{~nm}$ x $720 \mathrm{~nm}$, maximum load $=2000 \mu \mathrm{N}$ 1 color $\Leftrightarrow 1 \mathrm{~nm}$ height 


\section{FIGURE 4}

(a)

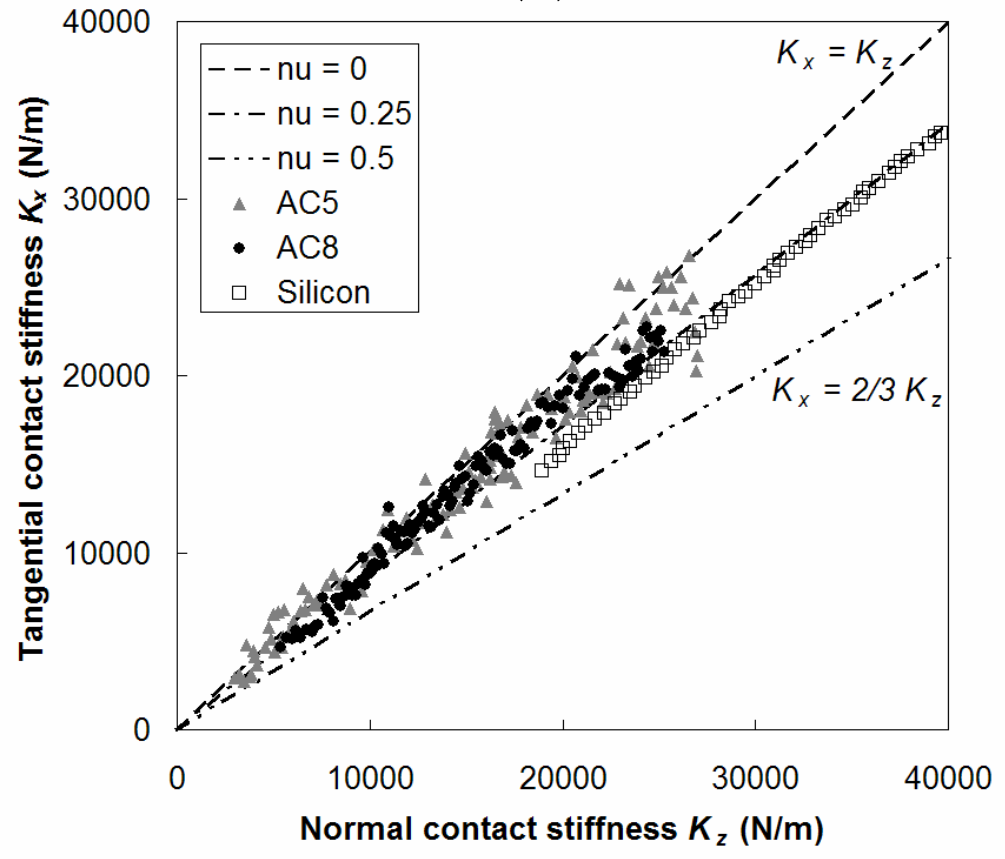

(b)

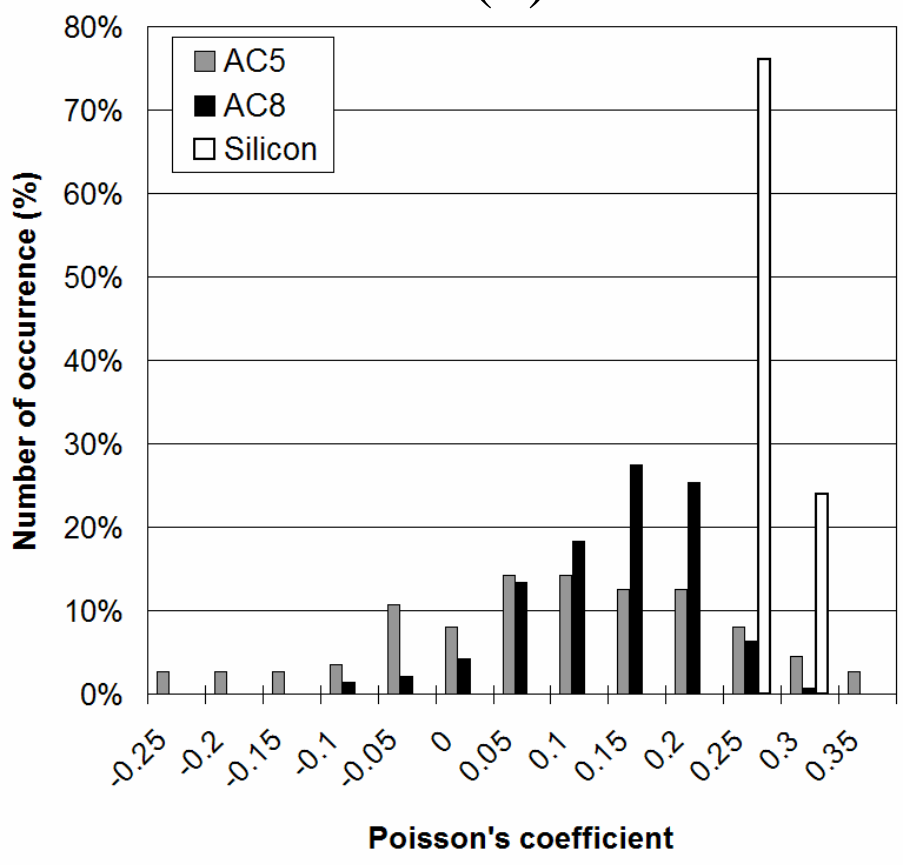




\section{FIGURE 5}

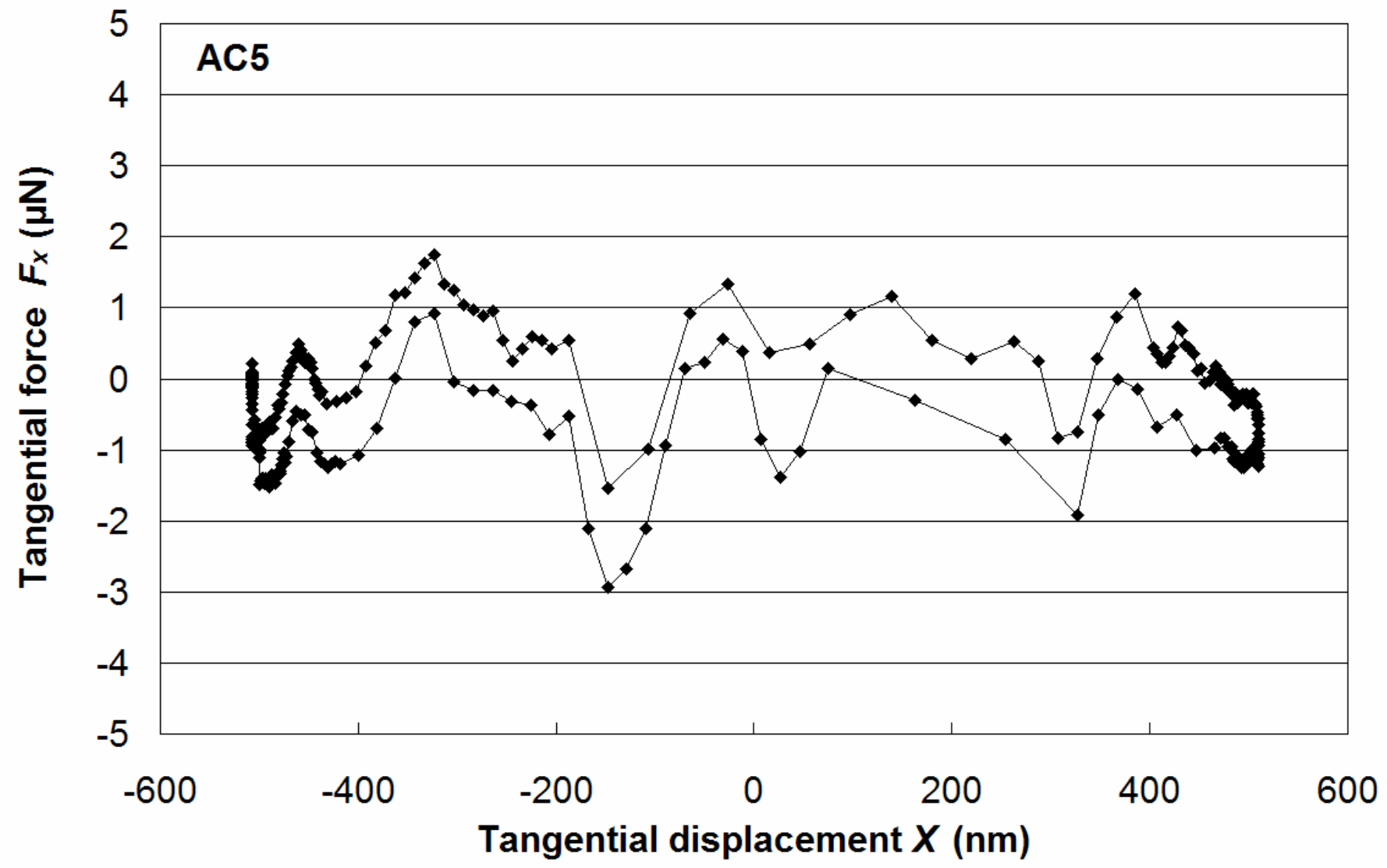


FIGURE 6

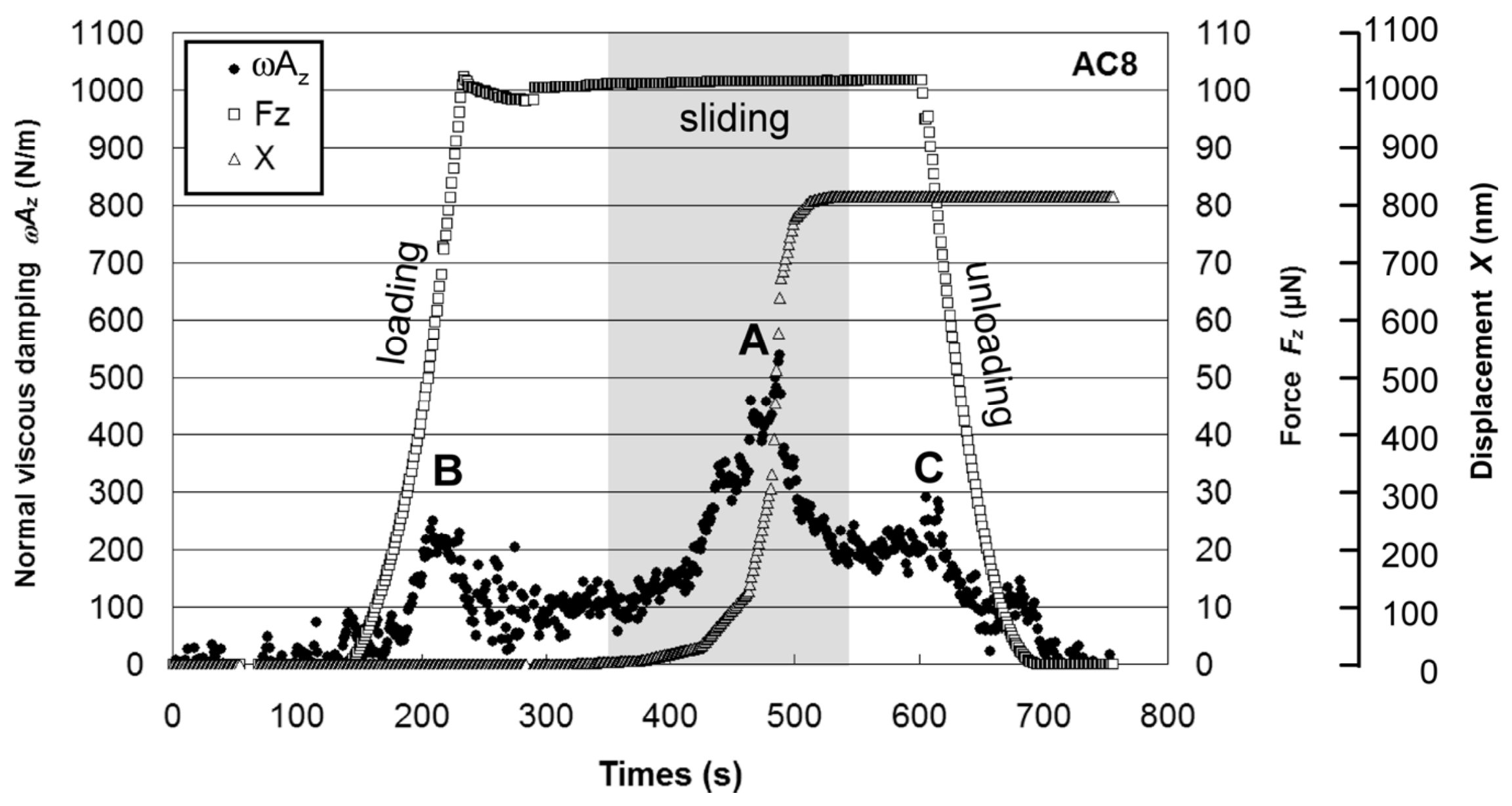

